(C) 2009 IEEE. Reprinted, with permission, from Daniel Franklin, Characterising the Behaviour of IEEE 802.11 Broadcast Transmissions in Ad Hoc Wireless LANs , Communications, 2009. ICC '09. IEEE International Conference on, June 2009. This material is posted here with permission of the IEEE. Such permission of the IEEE does not in any way imply IEEE endorsement of any of the University of Technology, Sydney's products or services. Internal or personal use of this material is permitted. However, permission to reprint/republish this material for advertising or promotional purposes or for creating new collective works for resale or redistribution must be obtained from the IEEE by writing to pubs-permissions@ieee.org. By choosing to view this document, you agree to all provisions of the copyright laws protecting it 


\title{
Characterising the Behaviour of IEEE 802.11 Broadcast Transmissions in Ad Hoc Wireless LANs
}

\author{
Jerry Chun-Ping Wang, Mehran Abolhasan, Daniel R. Franklin, Farzad Safaei \\ Telecommunication \& IT Research Institute \\ University of Wollongong, Wollongong, NSW 2522, Australia \\ \{jcpw942,mehrana,danielf,farzad\}@uow.edu.au
}

\begin{abstract}
This paper evaluates the performance of the IEEE 802.11 broadcast traffic under both saturation and nonsaturation conditions. It also highlights some important characteristics of IEEE 802.11 broadcast traffic as compare to corresponding unicast traffic. Further, it improves the accuracy of the existing broadcast saturation model proposed by Ma et al by considering the freezing of the backoff counter when channel is busy. Computer simulations are used to validate the accuracy of the model and demonstrate the importance of capturing the freezing of backoff counter in the analytical study of IEEE 802.11 broadcast.
\end{abstract}

Index Terms-IEEE 802.11 DCF, Broadcast, Non-Saturation Analysis

\section{INTRODUCTION}

Broadcasting is one of the essential communication techniques used in ad hoc networks. In particular, many ad hoc routing protocols rely heavily upon the MAC layer's broadcast service to discover neighbours and disseminate and maintain up-to-date routing information. Although the wireless medium is inherently broadcast in nature, there are a number of key differences between the handling of unicast and broadcast frames - for example, the RTS/CTS mechanism cannot be used for broadcast frames due to the one-to-many nature of broadcast traffic.

The study of IEEE 802.11 broadcast has been largely neglected in the past as broadcasts can be considered as a trivial component of a typical WLAN environment (since broadcast frames are mostly transmitted by the access point). Consequently, early analytical studies of the IEEE 802.11 distributed coordination function (DCF) mostly emphasised performance evaluation of unicast transmission. A two-dimensional Markov chain model has been developed by Bianchi [1] which can be used to evaluate the saturation throughput of IEEE 802.11 unicast traffic. Many subsequent studies extended Bianchi's model, for example, by including a model for the freezing of the backoff counter [2]-[4] which occurs when the channel is busy. Other studies have evaluated the performance of unicast transmission in a non-saturated network [5]-[7].

Given the importance of distributing updated network topology information throughout an ad-hoc network, there is a strong motivation for developing a better understanding of

This work is partly supported by the Desert Knowledge CRC (DK-CRC) under the joint DK-CRC / University of Wollongong (UoW) project "Sparse Ad hoc Network for Desert (SAND)". the behaviour of broadcast transmissions. Ma and Chen [8] realised that the existing Markov models for unicast traffic typically assume infinite retry limits, which is not appropriate for broadcast traffic. To address this shortcoming, they developed a one-dimensional Markov model which they used to evaluate the performance of broadcast traffic under saturated network conditions. Wang and Mahbub [9] have proposed a similar model, which also accounts for the freezing of the backoff process. While these models have been able to accurately predict the saturation performance of broadcast traffic, broadcast transmissions, in general, do not dominate a typical traffic mix. In fact, they usually comprise only a small portion of the total network load. Furthermore, most practically useful ad hoc networks do not operate under broadcast saturation conditions.

In this paper, an analytical study of IEEE 802.11 broadcast traffic with various network loads is presented. This model provides the following technical contributions:

- The one-dimensional Markov model developed in [8] is extended by considering the freezing of backoff counter and non-saturated process (Section III). The model is verified using computer simulations (Section IV).

- The performance of the IEEE 802.11 broadcast with various traffic loads and window sizes is evaluated and characterised (Section V).

- The non-saturation performance of broadcast traffic is compared with the corresponding unicast counterpart to highlight some important characteristics of broadcast transmission (Section VI).

- The saturation performance estimated by the existing model [8] and the extended model are compared, and it is shown that the existing model generally underestimates the saturation throughput due the absence of backoff counter freeze process (Section VII).

Before the performance of the broadcast transmission is evaluated in detail, Section II explains the operation of broadcast traffic as specified in the IEEE 802.11 protocol.

\section{IEEE 802.11 BROADCAST OPERATION}

The operation of IEEE 802.11 broadcast is formally defined in Section 9.2.7 of the IEEE 802.11 protocol specification [10]. Since the broadcast frame does not include a specific destination address, no RTS/CTS exchange can be used. Further, the recipients of the broadcast frame (if any) do not 
acknowledge the reception of broadcast frame - a broadcast simply constitutes a single-frame sequence. Because of this, the IEEE 802.11 standard mandates that the backoff window for broadcast traffic is always equal to the initial minimum backoff size (i.e. no binary exponential increase in window size in the event of a collision).

Further, the IEEE 802.11 DCF adopts both physical and virtual carrier sensing to detect the status of medium. When either operation indicates a busy medium, the medium is considered to be busy, and free otherwise. A station must stop decrementing the backoff counter if the medium appears to be busy. It is important to note that for broadcast transmission, although the broadcast frames can not provide virtual carrier sensing through exchange of RTS/CTS messages, the physical carrier sensing mechanism still applies. Any station that overhears any on-going transmission must stop backoff countdown until the medium becomes free.

\section{AnAlyticAl Model}

The analytical model presented in this paper is based on the non-saturated Markov process from [5], with the freezing of backoff counter adapted from [4], [9]. The network is assumed to be a single-hop ad hoc network with $n$ contending stations. The transmission environment is a two-ray propagation model with no hidden terminal or capture effects, so all packet losses are due to collisions. It is assumed that each station can buffer exactly one packet at a time, and that the arrival process of broadcast traffic is Poisson with mean arrival rate of $\lambda$.

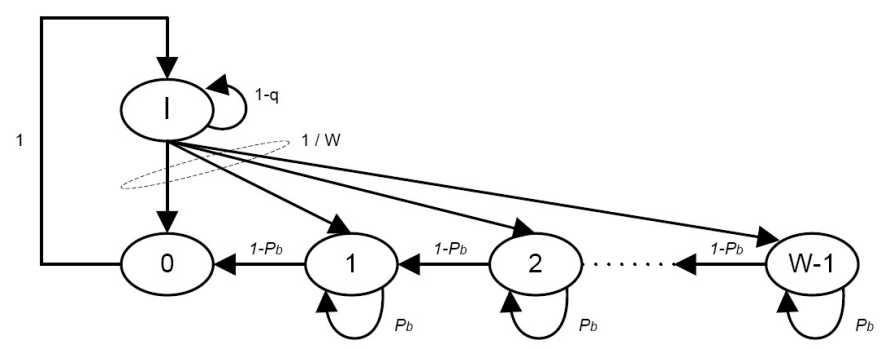

Fig. 1. State transition diagram for non-saturated Broadcast Model

\begin{tabular}{c|c}
\hline Notation & Description \\
\hline$n$ & Number of stations \\
$P_{b}$ & Channel busy probability \\
$q$ & Packet arrival probability \\
$W$ & Contention window size \\
\hline
\end{tabular}

TABLE I

NOTATION USED FOR ANALYTICAL MODEL

Figure 1 shows the state transition diagram used to model the IEEE 802.11 broadcast mechanism, and Table I summarises the notation that will be used in the model. As can be seen in Figure 1, the proposed one-dimensional Markov process contains $W$ backoff states (denoted $0 \ldots W-1$ ) and an additional idle state (denoted $I$ ). By considering the probability of packet arrival and the probability that the channel is busy, the system has the following non-null one-step transition probabilities:

$$
\left\{\begin{array}{l}
P\{I \mid I\}=1-q \\
P\{k \mid I\}=q / W \\
P\{k \mid k+1\}=1-p_{b} \\
P\{k \mid k\}=p_{b} \\
P\{I \mid 0\}=1
\end{array}\right.
$$

Adopting the same convention as Ma et al [8], let $b_{k}(k \in$ $[0, W-1])$ be the stationary distribution of backoff states, and let $b_{I}$ be the stationary distribution of the idle state. Through chain regularity, the broadcast process can be reduced to the following closed form solutions:

$$
\begin{gathered}
b_{I}=\frac{1}{q} b_{0} \\
b_{k}=\frac{(W-k) q}{2\left(1-P_{b}\right)} b_{I}=\frac{(W-k)}{2\left(1-P_{b}\right)} b_{0} \quad k \in[1, W-1] \\
b_{I}+b_{0}+\sum_{k=1}^{W-1} b_{k}=1
\end{gathered}
$$

Since a station is only allowed to transmit when the backoff counter reaches zero, the probability of transmission $\tau$ equals $b_{0}$. From Equations (2), (3), and (4), $\tau$ can be derived in the form of two variables $q$ and $P_{b}$ (defined below):

$$
\tau=b_{0}=\left(\frac{1}{q}+1+\frac{(W-1)}{2\left(1-P_{b}\right)}\right)^{-1}
$$

Given the transmission probability $\tau$, the throughput can be obtained following the classic roadmap of Bianchi's analysis [1]. However, the proposed model also includes the freezing of backoff process from [4], [9] and non-saturation process from [5]. Thus, we combine and reproduce the equations from the existing references for the clarity of derivation and ease of reference.

Let $P_{b}$ be the probability that the channel is in use, and $P_{s}$ be the successful transmission probability, they can be expressed as:

$$
\begin{aligned}
& P_{b}=1-(1-\tau)^{n} \\
& P_{s}=n \tau(1-\tau)^{n-1}
\end{aligned}
$$

According to Bianchi [1], the interval between two consecutive backoff states is represented by a single timeslot period known as the virtual timeslot. The virtual timeslot includes either an empty slot, a collision or a successful transmission, and its average length is equal to

$$
\text { SlotTime }=\left(1-P_{b}\right) \sigma+P_{b} T
$$

where $\sigma$ represents the duration of an empty slot. Since the broadcast does not employ the RTS/CTS mechanism or acknowledgement (ACK), the cost of successful and unsuccessful transmission is identical. Let $H=$ MAC header + 
PHY header (i.e. the size of header), and $E[P]$ be the data payload size. Assuming the system has channel bitrate $R$ and propagation delay $\delta$, the time required for channel access $T$ is given by:

$$
T=\frac{H+E[P]}{R}+D I F S+\delta
$$

The packet transmission probability $q$ determines the offered load that a station can inject into the network. The model assumes the packet arrival process is Poisson with an arrival rate of $\lambda$. The packet arrival probability $q$ can now be expressed as:

$$
q=1-e^{-\lambda T i m e S l o t}
$$

Equations (5), (6) and (9) form a non-linear system of equations in three unknowns $\tau, P_{b}$ and $q$, which can be numerically solved through non-linear optimisation. Once the system is solved and the numerical values are obtained, the throughput can be calculated as the amount of successful bits transmitted over a virtual timeslot, as given by

$$
S=\frac{P_{s} E[P]}{\text { SlotTime }}
$$

\section{Model VAlidation}

In order to validate the proposed analytical model, a series of simulations have been conducted using a discrete-event network simulator (Qualnet 4.0) and the results are compared against those obtained from the analytical model.

The simulation environment consists of $n+1$ stations randomly distributed across a square flat region of $100 \mathrm{~m}$ x $100 \mathrm{~m}$. Each station has a single $802.11 \mathrm{~b}$ wireless network interface and an omni-directional antenna positioned 1.5 meters above the ground. The RF channel is represented by a two-ray propagation model, and the maximum data bit-rate set at 1 Mbps. Under these conditions, each station has maximum transmission range approximately $450 \mathrm{~m}$. Therefore, all nodes are within one hop of each other.

The simulation employs Qualnet's MAC_DOT11 library as the MAC protocol, and the routing function is switched off to ensure the traffic is only generated from the application layer. The important MAC parameters used for the analytical model and simulations follow those specified for the DSSS (Direct Sequence Spread Spectrum) PHY layer (Section 18.3.3 in [10]), which is shown in Table II. Apart from aforementioned parameters, the remaining of parameters adopt the default values specified by Qualnet simulator.

The broadcast traffic is generated by Qualnet's traffic generator (Traffic-Gen) which transmits broadcast frames with fixed payload size (8184 bits). Further, the packets are generated using a Poisson arrival with mean arrival rate $\lambda$. In the simulation, there are $\mathrm{n}$ stations, each acts as a source of one UDP broadcast flow. We also include an additional station to serve as a sink of all broadcast flows. The data representing the simulation results are those collected from the sink node. For the accuracy of simulation results, each simulation was run

\begin{tabular}{c|c}
\multicolumn{3}{c}{ System Parameters } \\
\hline Packet Payload $(E[P])$ & 8184 bits \\
MAC Header & 272 bits \\
PHY Header & 128 bits \\
Channel Bit Rate $(R)$ & $1 \mathrm{Mbits} / \mathrm{s}$ \\
Propagation Delay $(\delta)$ & $1 \mu \mathrm{s}$ \\
Slot Time $(\sigma)$ & $20 \mu \mathrm{s}$ \\
SIFS & $10 \mu \mathrm{s}$ \\
DIFS & $50 \mu \mathrm{s}$ \\
\hline
\end{tabular}

TABLE II

MAC PARAMETERS USED FOR ANALYTICAL MODEL AND SIMULATION

for 300 seconds and all simulation results shown have been obtained from the average of at least 15 independent runs, with more than $95 \%$ of result being within $1 \%$ of the average value for all simulation results.

\section{Non-Saturation Throughput Analysis}

In this section, the throughput of IEEE 802.11 broadcast traffic under non-saturation conditions with different offered loads is presented. The offered load used in this paper is defined as the average bit rate being transmitted through the wireless network interface divided by the channel bit-rate (i.e. $\lambda E[P] / R)$.

Figure 2 shows the non-saturated throughput for broadcast traffic with different offered load levels. In this experiment, both the analytical model and simulation assume the contention window size is set to $32(W=32)$, and evaluate the performance with various numbers of operational stations. As can be seen from Figure 2, the predicted throughput (lines) accurately predicts the simulated throughput (markers). Both analytic and simulated results illustrate several important characteristics of IEEE 802.11 broadcast traffic. In particular, the throughput exhibits a linear relationship with offered load until the optimal throughput is reached. The throughput then declines and the network enters a state of saturation. The throughput reduction beyond the point of saturation is more pronounced for networks with a larger number of contending stations.

Figure 3 examines the impact of various window sizes with 10 stations contending for broadcast access. According to the Figure, the analytical model provides a good match with the simulations over most traffic levels. Regardless of the size of the contention window, it can be seen that all three results have demonstrated almost identical linear growth when the network load is low; the performance of three window sizes only begins to differ when the throughput reaches its maximum. The smallest window size (i.e. $W=16$ ) suffers the greatest reduction in throughput as the network becomes more saturated, whereas larger windows maintain a relatively constant throughput even when network is under high load. Clearly, the smaller window size does not cope well with network contention under heavy traffic conditions. Therefore, the throughput performance of IEEE 802.11 broadcast traffic is greatly affected by the contention window size under saturation conditions. 


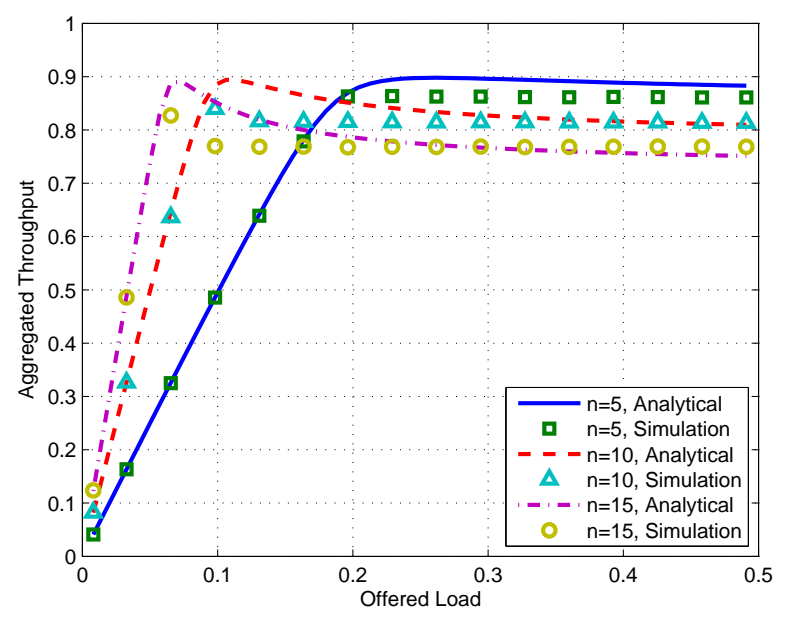

Fig. 2. Aggregated throughput versus offered load with fixed window size $(\mathrm{W}=32)$

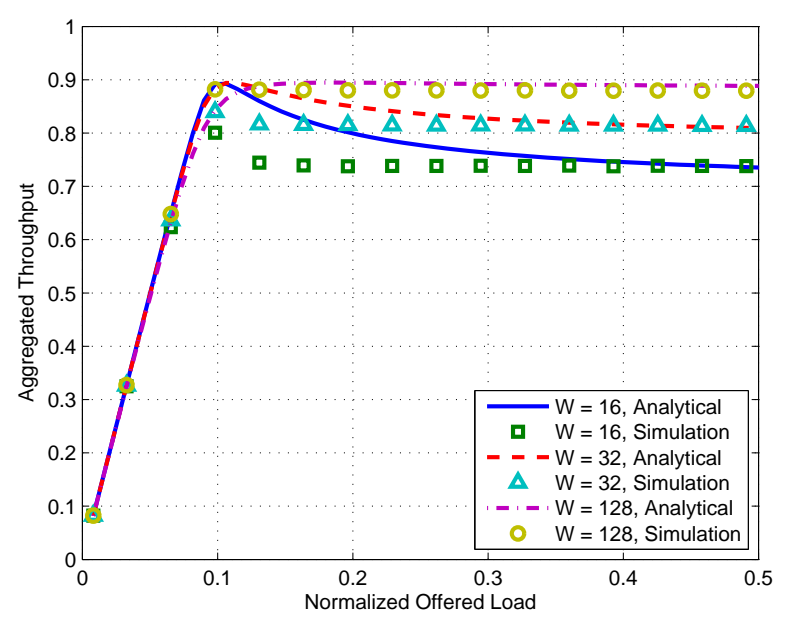

Fig. 3. Aggregated throughput versus offered load with fixed number of stations $(n=10)$

\section{COMPARISON WITH UNICAST TRANSMISSIONS}

To highlight the important characteristics of broadcast transmission with different load, this section presents the head-tohead comparison of the two traffic classes with diffrent offered load. We adopt the non-saturation performance of unicast transmission from [5] along with the freezing of backoff process from [4]. In this comparison, we assume the unicast transmission follows the same parameters used for broadcast transmission with the inclusion of retry limit of 5 for unicast transmission, so the unicast transmission will continuously expand its contention window upon each collision until the transmission is successful or the retry limit is reached. The initial contention window size is set to 32 for both traffic classes.

Figure 4 illustrates the non-saturation throughput of unicast and broadcast transmissions with various offered load. The

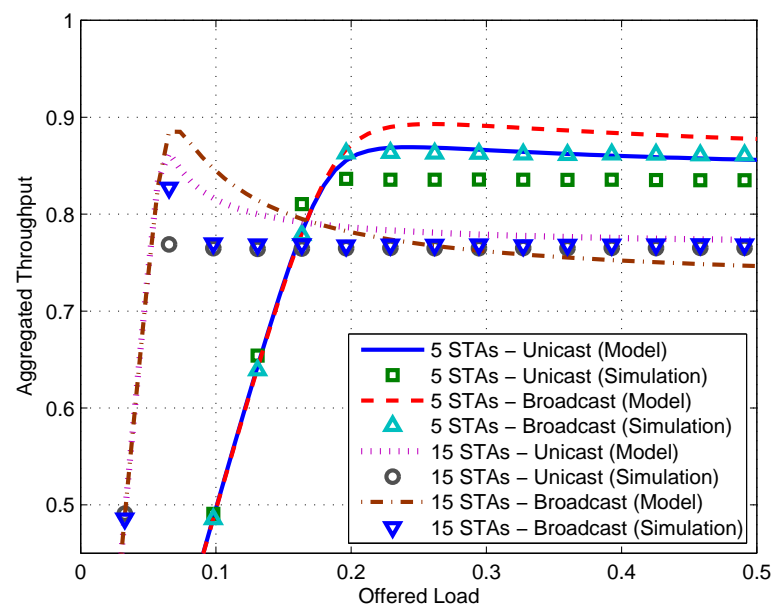

Fig. 4. Comparisons with unicast transmissions

simulation results closely match the analytical results for both traffic classes. By comparing the results of the broadcast transmission with its corresponding unicast component, it can be seen that the broadcast transmission exhibits identical linear relationship between throughput and offered load as the unicast counterpart prior to the saturation. From the Figure, it is notable that the broadcast transmission maintains higher optimal throughput than the unicast transmission. The broadcast transmission on one hand takes advantage of lower transmission overhead (i.e. no acknowledgement required for broadcast transmission) when the network experiences less packet collisions prior saturation. On the other hand, as the network becomes saturated and the packet collisions appear to be more frequent, the transmission overhead in addition to collisions creates more delays. Therefore, the advantage of lower transmission overhead could be diminished as the network contention scales up.

Since the broadcast transmission fundamentally differs from unicast transmission by the absence of binary exponential backoff, the broandcast transmission has the trade-off of suffering higher throughput degradation beyond the point of saturation. However, it should be noted that the binary exponential backoff can only be effective upon collisions. Therefore, the throughput degradation is subject to the scale of contention. For instance, Figure 4 demonstrates that the broadcast transmissions maintain higher throughput than unicast transmissions at all time under low network contention. As there are more stations competing for access (i.e. 15 stations) the broadcast throughput could drastically deteriorate from optimal throughput which is higher than unicast transmission to the saturation throughput that is lower than the unicast transmissions. In other word, the unicast transmission is more resilient under higher network contention, whereas the broadcast transmission is more effective under lower network contention. 


\section{REMARKS ON SATURATION THROUGHPUT}

Figures 2 and 3 demonstrate that prior to the point of saturation, throughput increases linearly with offered load, as expected. Beyond the critical point at which maximum throughput is attained, according to the new analytic model, throughput converges to an asymptotic saturation level, which depends on the number of nodes contending for access. This saturation level may be evaluated by considering what happens as the traffic load tends to infinity.

Let $q \rightarrow 1$, such that there is always a packet ready to send upon the completion of a broadcast transmission. The saturated transmission probability $\tau^{s a t}$ can be redefined as

$\tau^{\text {sat }}=\lim _{q \rightarrow 1}\left(\frac{1}{q}+1+\frac{W-1}{2\left(1-P_{b}\right)}\right)^{-1}=\left(2+\frac{W-1}{2\left(1-P_{b}\right)}\right)^{-1}$

To obtain the saturated throughput from the proposed analytical model, a non-linear optimisation technique (i.e. Newton's Method) is used to iteratively solve the non-linear system of Equations (11) and (6). In order to provide the accurate evaluation of the saturation performance, the simulation results are also included, with the traffic generator modified such that a packet is always available for transmission. The same protocol parameters were used as before.

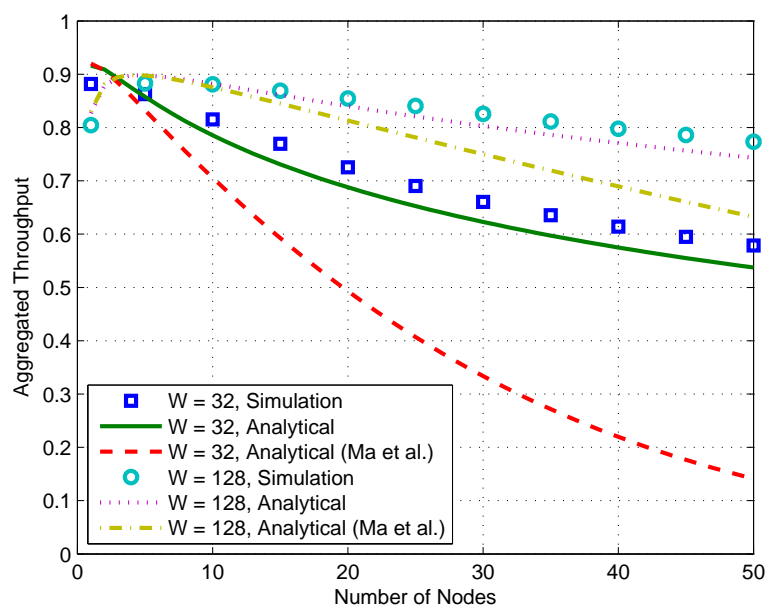

Fig. 5. Comparisons of saturation throughput

The numerical results for the saturation throughput obtained from simulations, the proposed analytic model and the analytic model proposed by Ma et al [8] are plotted in Figure 5. The new model clearly provides a much more accurate prediction of saturation throughput for IEEE 802.11 broadcast traffic as compared to the earlier model, which significantly underestimates saturation throughput, especially when a smaller window size is used with a large number of contending stations.

The new model principally differs from its predecessor by the inclusion of the freezing of backoff counter. Previous models have neglected the physical carrier sensing function, and continuously decrement the backoff counter even when channel is busy. This increases the probability of broadcast collisions as more stations are transmitting, which subsequently results in a much lower saturation throughput. Therefore, the inclusion of the freezing of backoff counter is shown to be critical in the accurate analytical evaluation of the IEEE 802.11 broadcast, and this new model represents a significant enhancement of existing analytic work in this area.

\section{CONCLUSION}

In this paper, the non-saturated and saturated performance of the IEEE 802.11 broadcast is investigated both analytically and through computer simulation. A new model has been developed using an extended Markov model with backoff counter freezing, which has been shown to accurately predict network performance over a wide range of traffic loads, particularly when the network approaches saturation. The comparison with unicast traffic has shown the unique characteristics of broadcast traffic. On one hand, broadcast traffic achieves higher optimal throughput than its unicast counterpart under low traffic load. On the other hand, as the traffic load increases, the performance of broadcast may deteriorate at much faster rate and result in lower throughput than unicast traffic depending on the scale of network contention. Future work will include an extension of the broadcast model to support unicast traffic, to further examine the performance of networks with mixed unicast and broadcast traffic.

\section{REFERENCES}

[1] G. Bianchi, "Performance analysis of the IEEE 802.11 distributed coordination function," IEEE Journal on Selected Areas in Communications, vol. 18 , no. 3, pp. 535-547, March 2000.

[2] E. Ziouva and T. Antonakopoulos, "CSMA/CA performance under high traffic conditions: throughput and delay analysis," Computer Communications, vol. 25, no. 3, pp. 313-321, February 2002. [Online]. Available: http://dx.doi.org/10.1016/S0140-3664(01)00369-3

[3] C. H. Foh and J. W. Tantra, "Comments on IEEE 802.11 saturation throughput analysis with freezing of backoff counters," IEEE Communications Letters, vol. 9, no. 2, pp. 130-132, February 2005.

[4] S. J. Kim, H. Y. Hwang, J. K. Kwon, and D. K. Sung, "Saturation performance analysis of IEEE 802.11 WLAN under the assumption of no consecutive transmissions," IEICE Transactions on Communications, vol. E90-B, no. 3, pp. 700-703, March 2007.

[5] C. Xu and Z. Yang, "Non-saturated throughput analysis of IEEE 802.11 ad hoc networks," IEICE Transactions on Information and Systems, vol. E89-D, no. 5, pp. 1676-1678, May 2006.

[6] D. Malone, K. Duffy, and D. Leith, "Modeling the 802.11 distributed coordination function in nonsaturated heterogeneous conditions," IEEE/ACM Transactions on Networking, vol. 15, no. 1, pp. 159172, 2007.

[7] N. T. Dao and R. A. Malaney, "A new markov model for nonsaturated 802.11 networks," in Proceedings of 5th IEEE Consumer Communications and Networking Conference (CCNC 2008), January 2008, pp. 420-424.

[8] X. Ma and X. Chen, "Performance analysis of IEEE 802.11 broadcast scheme in ad hoc wireless lans," to appear in IEEE Transactions on Vehicular Technology, 2008.

[9] Z. Wang and M. Hassan, "Analytical evaluation of the 802.11 wireless broadcast under saturated conditions," School of Computer Science and Engineering, University of New South Wales, Tech. Rep. UNSW-CSETR-0801, January 2008

[10] IEEE, IEEE Std. 802.11-2007, Part 11: Wireless LAN Medium Access Control (MAC) and Physical Layer (PHY) specifications, 2007. 